\title{
Interaction between a Macrocrack and a Cluster of Microcracks by Muskhelishvili's Complex Potential Method
}

\author{
Xu Li, Xiaotao Li, Hongda Yang, and Xiaoyu Jiang \\ Applied Mechanics and Structure Safety Key Laboratory of Sichuan Province, School of Mechanics and Engineering, \\ Southwest Jiaotong University, Chengdu 610031, China
}

Correspondence should be addressed to Xiaoyu Jiang; xiaoyujiang8@sina.com

Received 22 July 2018; Accepted 17 September 2018; Published 9 October 2018

Academic Editor: Paolo Lonetti

Copyright (C) $2018 \mathrm{Xu} \mathrm{Li} \mathrm{et} \mathrm{al.} \mathrm{This} \mathrm{is} \mathrm{an} \mathrm{open} \mathrm{access} \mathrm{article} \mathrm{distributed} \mathrm{under} \mathrm{the} \mathrm{Creative} \mathrm{Commons} \mathrm{Attribution} \mathrm{License,} \mathrm{which}$ permits unrestricted use, distribution, and reproduction in any medium, provided the original work is properly cited.

The interaction between a macrocrack and a cluster of microcracks has been investigated based on Muskhelishvili's complex potential method. A step-by-step subproblem procedure is used to satisfy the stress boundary conditions on each crack surface. The interactions between a cluster of microcracks and a macrocrack and the interaction among microcracks are analyzed. Three damage configurations as chained, reverse-chained, and randomly distributed microcracks have been designed to simulate the damage around the macrocrack tip. The solution of an infinite elastic plane containing a macrocrack and a cluster of microcracks is presented for the plane subjected to a uniform tensile load. The stress intensity factor (SIF) at the macrocrack tip and the microcrack tips is obtained. The results show that the inclination angle of the microcrack and the distance between the macrocrack and microcracks have a great influence on SIF. When the inclination angle is small, the SIF at microcrack tips may be larger than other inclination angles. These results are helpful to analyze the fracture or damage behaviors of materials.

\section{Introduction}

Many experimental investigations demonstrate that profuse microcrack propagations can be observed during the evolution of fracture process in the high-stress concentration regions, as well at the tip of the macrocrack [1-4]. The microcrack has a great effect on SIF at the macrocrack tip. Thus, the interaction between a macrocrack and multiple microcracks is a topical problem in elastic theory and fracture mechanics.

Many results concerning the interaction of macrocrack and arrays of microcracks were obtained using various numerical methods such as serious expansions, boundary element methods, and collocation methods. Crack propagation mechanisms can be developed if the basic question whether the microcracks enhance or suppress the macrocrack propagation is answered. The pseudotractions method has been developed by many studies [5-9] to investigate the interaction problem between main crack and microcracks. The core idea of this method was that a superposition of a number of subproblems each contains one crack loaded by unknown tractions. Kachanov [10-12] proposed the interaction matrix between cracks in the theory. He also replaced the actual traction by average traction. Based on small parameter method, Tamuzs et al. $[13,14]$ investigated the interaction of a macrocrack with a cluster of cracks; Petrova et al. [1517] effectively applied it in thermal fracture of a functionally graded/homogeneous bimaterials with series of cracks. Hoagland [18] and Ismail [19] studied the process of the nucleation and interaction of microcracks near a macrocrack tip by using the complex potential method and the theory of dislocation. Yan Xiangqiao [20,21] presented a numerical approach for the interaction problem of macrocrack with microcrack in an infinite elastic plane. Based on the principle of superposition and a flattening method, Xia Xiaozhou et al. [22] analyzed the interaction between cracks and effect of microcrack zone on main crack tip. Rafiee [23] and Seelig [24] investigated the interaction between cracks and branching of fast running cracks.

However, microcracks are mainly arrays in parallel and collinear in previous studies. In fact, the formation of microcracks is random, and randomly distributed microcracks in front of a macrocrack tip are usually used to model the material damage in some materials such as metals [25-27]. In this paper, three kinds of damage configurations as chained, reverse-chained, and randomly distributed microcracks have 
been designed to simulate the damage around the macrocrack tip.

The outline of this paper is as follows. In Section 2, a high efficient method based on Muskhelishvili's complex potential method is presented to study the interaction between a finite macrocrack and a cluster of microcracks and the interaction among microcracks. A step-by-step subproblem procedure is used to satisfy the boundary conditions on each crack surface. The solution of an infinite plane that contains a macrocrack and arbitrary located and oriented microcracks is presented. In Section 3, numerical examples are employed to illustrate the accuracy of the proposed method. The influence of three kinds of arrangements of microcracks on SIF in the macrocrack tip is analyzed. In Section 4, some conclusions are drawn.

\section{Formulation}

2.1. Problem Description and Solution Scheme. The problem in terms of an infinite elastic plane containing a macrocrack and randomly distributed microcracks under remote uniform traction $T$ is considered, as shown in Figure 1. In order to a convenient statement, the microcrack from 1 to $k(k=$ $2, \ldots, j, \ldots, n)$ is called microcrack 1 to microcrack $k$ in order. In Figure 1, oxy is the global coordinate system with the origin at the center of the macrocrack. $o_{k} x_{k} y_{k}(k=1, \ldots, j, \ldots, n)$ is the local coordinate system with the origin at the center of microcrack $k$.

The problem in Figure 1 can be replaced by a series of subproblems, as shown in Figure 2. Each subproblem is independent.

With reference to Figure $2, d_{k}(k=1, \ldots, j, \ldots, n)$ is the distance between the macrocrack tip and the center of microcrack $k ; \beta_{k}$ is the inclination angle to the $x$-axis of microcrack $k ; \theta_{k}$ is the direction with respect to the macrocrack tip. In addition, $a$ is the half-length of the macrocrack, and $a_{k}$ is the half-length of microcrack $k$.

The subproblems are as follows: In subproblem (i), an infinite plane containing a finite macrocrack, without the microcrack, subjected to uniaxial tension, refer to Figure 2(a). In subproblem (ii), an infinite plane containing an arbitrarily oriented microcrack, without the macrocrack, subjected to the tractions obtained in subproblem (i) but opposite in sign. There are $\mathrm{n}$ microcracks corresponding to $\mathrm{n}$ subproblems in subproblem (ii), refer to Figure 2(b). In subproblem (iii), an infinite plane containing a finite macrocrack, without the microcrack, subjected to the tractions obtained in subproblem (ii) but opposite in sign, refer to Figure 2(c). Subproblem (iv) and subproblem (v) may be obtained through a similar process of subproblem (ii) and subproblem (iii) and so on.

It should be noted that the original problem is under remote uniform traction $T$, as shown in Figure 1. The stress boundary condition on each crack surface is traction free. The stress boundary condition is satisfied by the step-by-step subproblems. For example, the stresses along the line which is the location of microcrack $j$ in subproblem (i) are obtained. Then the opposite stresses are applied to the crack surface of microcrack $j$ in subproblem (ii). At this time, the stresses on

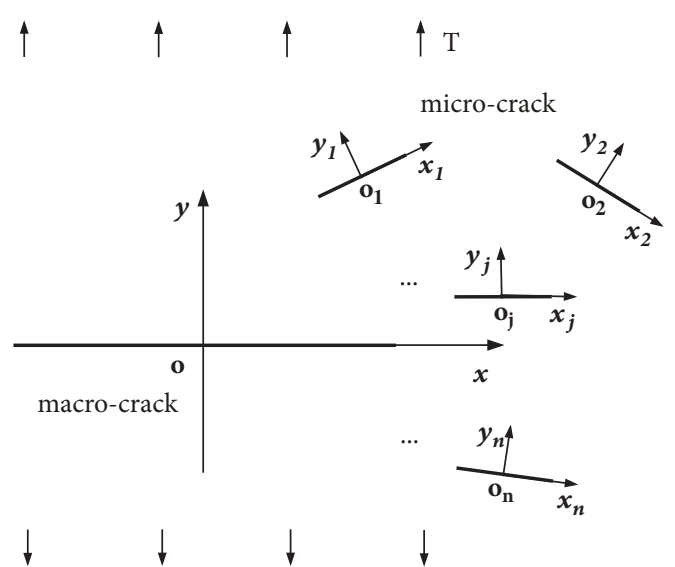

FIGURE 1: An infinite plane containing a macrocrack and multiple microcracks under mode I load.

the crack surface of microcrack $j$ are zero. But the stresses on the crack surface of microcrack $j$ are not zero because of the affection of other microcracks in subproblem (ii). And the nonzero value of stress on the crack surface of microcrack $j$ is a small order of magnitude. The nonzero value of stress on the crack surface of microcrack $j$ is added to the stress from subproblem (iii), and then the resultant stress (opposite in sign) is applied to the microcrack $j$ in subproblem (iv), and so on. In the end, the stress boundary condition of traction free on each crack surface is satisfied.

2.2. Mathematical Formulation of Subproblems. For plane elasticity, the stress fields of a single crack in the center of an infinite plane can be expressed in terms of two complex potentials $\Phi(z)$ and $\Psi(z)$ as [28]

$$
\begin{aligned}
\sigma_{x x}+\sigma_{y y} & =2\left\{\Phi^{\prime}(z)+\overline{\Phi^{\prime}}(z)\right\} \\
\sigma_{x x}-\sigma_{y y}+2 i \tau_{x y} & =2\left\{\bar{z} \Phi^{\prime \prime}(z)+\Psi^{\prime}(z)\right\}
\end{aligned}
$$

where $\mathrm{z}=x+i y ; \sigma_{x x}$ denotes the normal stress in the horizontal direction; $\sigma_{y y}$ denotes the normal stress in the vertical direction; $\tau_{x y}$ denotes the shear stress; $\kappa=3-4 \nu$ for plane strain, $\kappa=(3-v) /(1+\nu)$ for plane stress; $\mu$ is shear modulus of the material; $v$ is Poisson's ratio; $\left({ }^{\prime}\right)$ and $\left({ }^{\prime \prime}\right)$ are the first derivative and the second derivative of $z$, respectively.

2.2.1. Subproblem (i): The Macrocrack. In subproblem (i), the corresponding stress functions may be given by [28]

$$
\begin{aligned}
& \Phi(z)=\frac{T}{4}\left(2 \sqrt{z^{2}-a^{2}}-z\right) \\
& \Psi(z)=\frac{T}{2}\left(z-\frac{a^{2}}{\sqrt{z^{2}-a^{2}}}\right)
\end{aligned}
$$



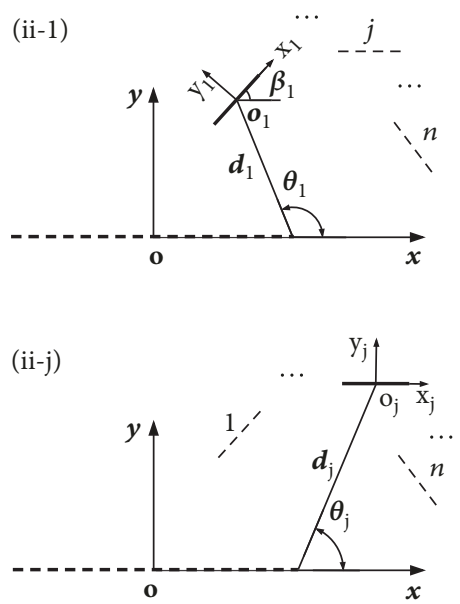

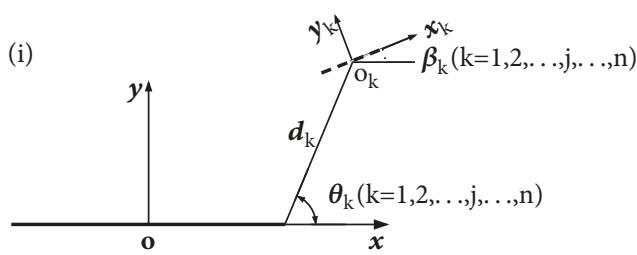

(a) Subproblem (i)

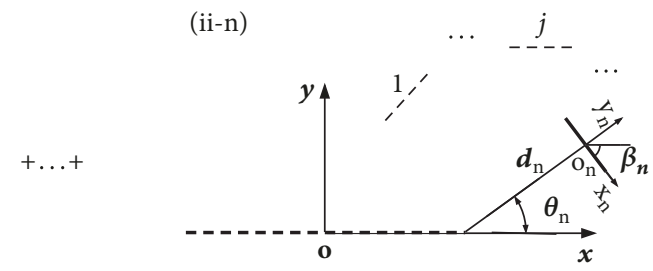

(b) Subproblem (ii)

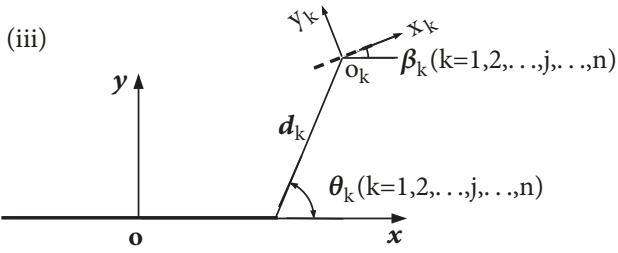

(c) Subproblem (iii)

FIGURE 2: The transformation of the original problem into a series of subproblems.

where $T$ is the applied loading as shown in Figure 1. Substituting (2) into (1), the well-known stress fields near the macrocrack tip may be obtained

$$
\begin{aligned}
& \sigma_{x x}=T \operatorname{Re}\left(\frac{z}{\sqrt{z^{2}-a^{2}}}-\frac{i a^{2} y}{\sqrt{\left(z^{2}-a^{2}\right)^{3}}}-1\right) \\
& \sigma_{y y}=T \operatorname{Re}\left(\frac{z}{\sqrt{z^{2}-a^{2}}}+\frac{i a^{2} y}{\sqrt{\left(z^{2}-a^{2}\right)^{3}}}\right) \\
& \tau_{x y}=T \operatorname{Im}\left(\frac{i a^{2} y}{\sqrt{\left(z^{2}-a^{2}\right)^{3}}}\right)
\end{aligned}
$$

For the nonexistent microcrack $k$, substituting the position parameters $d_{k}, \theta_{k}, \beta_{k}$, and $a_{k}$ of the microcrack into (3), the stresses $\sigma_{x x(k)}^{(i)}, \sigma_{y y(k)}^{(i)}$, and $\tau_{x y(k)}^{(i)}$ along the position of the microcrack in the global coordinate system oxy are obtained, the superscript (i) on the stress $\sigma$ and $\tau$ denotes the results in subproblem (i), and the subscript $(k)$ denotes microcrack $k$.

By coordinate transformation, the normal and tangential stresses along the position of microcrack $k$ in the local coordinate system $o_{k} x_{k} y_{k}$ may be obtained by

$$
\begin{aligned}
\sigma_{N(k)}^{M I(i)}= & \frac{\sigma_{x x(k)}^{(i)}+\sigma_{y y(k)}^{(i)}}{2}+\frac{\sigma_{x x(k)}^{(i)}-\sigma_{y y(k)}^{(i)}}{2} \cos 2 \beta_{k} \\
& -\tau_{x y(k)}^{(i)} \sin 2 \beta_{k} \\
\tau_{T(k)}^{M I(i)}= & \frac{\sigma_{x x(k)}^{(i)}-\sigma_{y y(k)}^{(i)}}{2} \sin 2 \beta_{k}+\tau_{x y(k)}^{(i)} \cos 2 \beta_{k} \\
& (k=1, \ldots, j, \ldots, n)
\end{aligned}
$$

where the subscripts $N$ and $T$ denote the normal and tangential direction of the crack surface, respectively.

2.2.2. Subproblem (ii): The Microcracks. In subproblem (ii), the crack surface is subjected to a nonuniform distributed load that is equal in magnitude and opposite in sign to the 


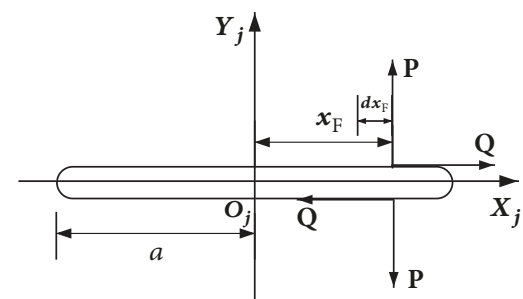

(a)

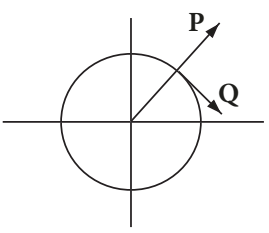

(b)

Figure 3: A crack subjected to concentrated forces. (a) $z$ - plane; (b) $\zeta$ - plane.

stresses obtained in subproblem (i). To solve subproblem (ii), it is necessary to introduce the problem shown in Figure 3. In the coordinate system $o_{j} x_{j} y_{j}$, a pair of concentrated forces $F=P+i Q$ acts at a point $z_{F}=x_{F}+i y_{F}$ of the crack surface in an infinite elastic plane, where $P$ is in the normal direction and $Q$ is in the tangential direction.

Then, introduce the mapping functions (see (5)), where $\zeta$ is the inverse function of $z, \zeta=\xi+i \eta$ is another complex variable, $m=1, R=a / 2$, and $a$ is the half-length of the crack. The crack surface in the $z$ - plane is transformed into a unit circle in the transformed $\zeta$-plane, and $z=\infty$ is corresponding to $\zeta=\infty$.

$$
\begin{aligned}
& z=\omega(\zeta)=R\left(\zeta+\frac{m}{\zeta}\right) \\
& \zeta=\omega^{-1}(z)=\frac{1}{a}\left(z \pm \sqrt{z^{2}-a^{2}}\right)
\end{aligned}
$$

Therefore, the stress functions of the concentrated forces applied to the upper and lower boundary of the crack will have the following forms [28]:

$$
\begin{gathered}
\Phi(\zeta)=\frac{F}{2 \pi i}\left\{-\frac{\zeta_{0}}{\zeta\left(\zeta_{0}^{2}-1\right)}-\frac{\kappa}{\kappa+1} \ln \zeta-\ln \left(\zeta_{0}-\zeta\right)\right. \\
\left.+\frac{\zeta_{0}^{2}\left[(a / 2)(\zeta+1 / \zeta)-z_{1}\right]}{(a / 2)\left(\zeta_{0}-\zeta\right)\left(\zeta_{0}^{2}-1\right)}\right\} \frac{i\left(\zeta_{0}^{2}-1\right)}{\zeta_{0}\left|\zeta_{0}-\bar{\zeta}_{0}\right|} \\
\Psi(\zeta)=\frac{F}{2 \pi i}\left\{-\frac{2 \zeta \zeta_{0}}{\left(\zeta^{2}-1\right)\left(\zeta_{0}^{2}-1\right)}+\frac{\ln \zeta}{\kappa+1}\right. \\
+\frac{2}{(\kappa+1)\left(\zeta^{2}-1\right)}-\frac{\zeta_{0}^{2}\left(\zeta^{2}+1\right)}{\left(\zeta^{2}-1\right)\left(\zeta_{0}^{2}-1\right)}-\ln \left(\zeta_{0}-\zeta\right) \\
\left.+\frac{\bar{z}_{1} \zeta_{0}^{2}}{(a / 2)\left(\zeta_{0}-\zeta\right)\left(\zeta_{0}^{2}-1\right)}\right\} \frac{i\left(\zeta_{0}^{2}-1\right)}{\zeta_{0}\left|\zeta_{0}-\bar{\zeta}_{0}\right|}
\end{gathered}
$$

With (5), the point $\zeta_{0}$ in $\zeta$ - planeis transformed to the point $z_{F}=(a / 2)\left(\zeta_{0}+1 / \zeta_{0}\right)$ in the $z$-plane, and the basic equation (6) may be rewritten in terms of the transformed $\Phi(z)$ and $\Psi(z)$.
Thus, in subproblem (ii), when a pair of concentrated forces $F$ is applied at the edge of the microcrack $j$ in the coordinate system $o_{j} x_{j} y_{j}$, where

$$
F=P+i Q=\left[-\sigma_{N(j)}^{M I(i)} d x_{F}\right]+i\left[-\tau_{T(j)}^{M I(i)} d x_{F}\right]
$$

$P$ and $Q$ are equal in magnitude and opposite in sign to the nonuniform stresses $\sigma_{N(j)}^{M I(i)}$ and $\tau_{T(j)}^{M I(i)}$ obtained in subproblem (i) along the line which is the presumed location of the microcrack $j$.

The stresses $\left(\sigma_{x x F}^{M A(i i)}, \sigma_{y y F}^{M A(i i)}, \tau_{x y F}^{M A(i i)}, \sigma_{x x F}^{M I(i i)}, \sigma_{y y F}^{M I(i i)}\right.$, and $\left.\tau_{x y F}^{M I(i i)}\right)$ along the position of the macrocrack and the microcrack $k(k=1,2, \ldots, n, k \neq j)$ can be calculated on the basis of (1), (5), (6), and (7). The superscript (ii) on the stress $\sigma$ and $\tau$ denotes the results in subproblem (ii), and the subscript $F$ denotes the results under concentrated forces $F$.

Then, when the nonuniform stresses act on the crack surface of the microcrack $j$ in an infinite plane, the stress fields of the macrocrack and microcrack $k j(k j=1, \ldots, n, k j \neq j)$ in the local coordinate system $o_{j} x_{j} y_{j}$ are obtained by

$$
\begin{aligned}
& \sigma_{x x(j)}^{M A(i i)}, \sigma_{y y(j)}^{M A(i i)}, \tau_{x y(j)}^{M A(i i)} \\
& =\int_{-a_{k}}^{a_{k}}\left(\sigma_{x x F}^{M A(i i)}, \sigma_{y y F}^{M A(i i)}, \tau_{x y F}^{M A(i i)}\right) d x_{F} \\
& \sigma_{x x(k j)}^{M I(i i)}, \sigma_{y y(k j)}^{M I(i i)}, \tau_{x y(k j)}^{M I(i i)} \\
& =\int_{-a_{k}}^{a_{k}}\left(\sigma_{x x F}^{M I(i i)}, \sigma_{y y F}^{M I(i i)}, \tau_{x y F}^{M I(i i)}\right) d x_{F} \\
& \sigma_{x x(k j)}^{M I(i i)}, \sigma_{y y(k j)}^{M I(i i)}, \tau_{x y(k j)}^{M I(i i)}=0 \quad(k j=1, \ldots, n, k j \neq j)
\end{aligned}
$$

For the macrocrack, the subscript $(j)$ denotes the effect of microcrack $j$ on the macrocrack. For the microcrack, the subscript $(k j)$ denotes the effect of microcrack $j$ on microcrack $k$. At this time, the stress on the crack surface of microcrack $j$ is zero because of the superposition of subproblem (i) and subproblem (ii).

Finally, transform the local coordinate system $o_{j} x_{j} y_{j}$ to the coordinate system of macrocrack $o x y$ and microcrack $o_{k j} x_{k j} y_{k j}(k j=1, \ldots, n)$, the solution $\left(\sigma_{N(j)}^{M A(i i)}, \tau_{T(j)}^{M A(i i)}, \sigma_{N(k j)}^{M I(i i)}\right.$, and $\left.\tau_{T(k j)}^{M I(i i)}\right)$ may be obtained for a nonuniform distributed 
load acting on the crack surface of microcrack $j$ in an infinite elastic plane. In the same way, we may use the formulas to derive the rest solutions in subproblem (ii).

There are $n$ microcracks corresponding to $n$ subproblems in subproblem (ii), the whole traction components of the macrocrack and microcracks are given by

$$
\begin{aligned}
& \sigma_{N}^{M A(i i)}=\sum_{j=1}^{n} \sigma_{N(j)}^{M A(i i)} \\
& \tau_{T}^{M A(i i)}=\sum_{j=1}^{n} \tau_{T(j)}^{M A(i i)} \\
& \sigma_{N(k)}^{M I(i i)}=\sum_{k j=1}^{n} \sigma_{N(k j)}^{M I(i i)} \\
& \tau_{T(k)}^{M I(i i)}=\sum_{k j=1}^{n} \tau_{T(k j)}^{M I(i i)}
\end{aligned}
$$

$$
(k=1, \ldots, n)
$$

It is shown from (10) that the stress on the crack surface of each microcrack is not zero because of the affection of other microcracks in subproblem (ii). But the nonzero value of stress on the crack surface of each microcrack is a small order of magnitude compared with the stresses $\sigma_{N(j)}^{M I(i)}$ and $\tau_{T(j)}^{M I(i)}$ obtained in subproblem (i).

2.2.3. Subproblem (iii): The Macrocrack. It is needed to study the problem of an infinite plane containing a macrocrack and without the microcracks. The crack surface is subjected to a nonuniform distributed load that is equal in magnitude and opposite in sign to the stresses in (9). It is used to solve the problem that the same method as described in subproblem (ii). When the concentrated forces $F$ acting at the upper and lower surface of the macrocrack, the stress fields $\sigma_{x x F}^{M I(i i i)}, \sigma_{y y F}^{M I(i i i)}$, and $\tau_{x y F}^{M I(i i i)}$ of the nonexist microcrack can be obtained; the expression of $F$ is

$$
F=P+i Q=\left[-\sigma_{N}^{M A(i i)} d x_{F}\right]+i\left[-\tau_{T}^{M A(i i)} d x_{F}\right]
$$

The stress components along the position of the microcrack are

$$
\begin{aligned}
& \sigma_{x x(k)}^{M I(i i i)}, \sigma_{y y(k)}^{M I(i i i)}, \tau_{x y(k)}^{M I(i i i)} \\
& \quad=\int_{-a}^{a}\left(\sigma_{x x F}^{M I(i i i)}, \sigma_{y y F}^{M I(i i i)}, \tau_{x y F}^{M I(i i i)}\right) d x_{F}
\end{aligned}
$$

$$
(k=1, \ldots, n)
$$

Finally, the normal and shear stresses $\sigma_{N(k)}^{M I(i i i)}$ and $\tau_{T(k)}^{M I(i i i)}$ in the coordinate system $o_{k} x_{k} y_{k}$ are obtained by the coordinate transformation from $o x y$ to $o_{k} x_{k} y_{k}(k=1, \ldots, n)$. In the end, the results $\sigma_{N(k)}^{M I(i i i)}$ and $\tau_{T(k)}^{M I(i i i)}$ may be obtained for microcracks in subproblem (iii).

2.2.4. Subproblem (iv): The Microcracks. $\sigma_{N(k)}^{M I(i i i)}$ and $\tau_{T(k)}^{M I(i i i)}$ are added with (10) to obtain the load on microcrack surface as $-\left(\sigma_{N(k)}^{M I(i i)}+\sigma_{N(k)}^{M I(i i i)}\right)$ and $-\left(\tau_{T(k)}^{M I(i i)}+\tau_{T(k)}^{M I(i i i)}\right)$ in subproblem (iv). The results $\sigma_{N}^{M A(i v)}, \tau_{T}^{M A(i v)}, \sigma_{N(k)}^{M I(i v)}$, and $\tau_{T(k)}^{M I(i v)}(k=$ $1, \ldots, n)$ may be obtained. The results are similar to (9) and (10).

In the following section, the numerical results for the interaction effect between the macrocrack and an array of microcracks and the interaction effect among microcracks have been presented.

By the aforementioned method, the interactions between the cracks are reflected in every subproblem, for example, in subproblem (i), the macrocrack effect on the microcrack; in subproblem (ii), the microcrack effect on the macrocrack and the interaction effect among the microcracks; in subproblem (iii), the macrocrack effect on the microcrack once again. Besides, the more the decomposition of the subproblems is, the higher the calculation accuracy can be obtained.

2.3. The Solution of $K_{I}$ and $K_{I I}$. As mentioned above, the traction-free boundary condition on the crack surface is satisfied by a step-by-step subproblem procedure. The results of each subproblem satisfy the fundamental equations of linear elastic mechanics independently.

In subproblem (i), the load on the macrocrack surface being $\sigma_{N}^{M A(i)}=-T$ and $\tau_{T}^{M A(i)}=0$, and the SIFs at the tip of the macrocrack are

$$
\begin{aligned}
& K_{I}^{M A(i)}=\int_{-a}^{a} \frac{-T}{\sqrt{\pi a}}\left(\frac{a+x}{a-x}\right)^{1 / 2} d x=T \sqrt{\pi a} \\
& K_{I I}^{M A(i)}=0
\end{aligned}
$$

In the step-by-step procedure, the loads applied on the macrocrack surface are $\sigma_{N}^{M A(i)}=-T$ and $\tau_{T}^{M A(i)}=0$ in subproblem (i), $\sigma_{N}^{M A(i i)}$ and $\tau_{T}^{M A(i i)}$ in subproblem (iii), $\sigma_{N}^{M A(i v)}$ and $\tau_{T}^{M A(i v)}$ in subproblem (v), etc. The SIFs at the tip of the macrocrack may be written as

$$
\begin{aligned}
& K_{I}^{M A}=T \sqrt{\pi a} \\
& \quad+\int_{-a}^{a} \frac{-\left[\sigma_{N}^{M A(i i)}+\sigma_{N}^{M A(i v)}+\sigma_{N}^{M A(v i)}+\ldots\right]}{\sqrt{\pi a}}\left(\frac{a+x}{a-x}\right)^{1 / 2} d x \\
& K_{I I}^{M A} \\
& \quad=\int_{-a}^{a} \frac{-\left[\tau_{T}^{M A(i i)}+\tau_{T}^{M A(i v)}+\tau_{T}^{M A(v i)}+\ldots\right]}{\sqrt{\pi a}}\left(\frac{a+x}{a-x}\right)^{1 / 2} d x
\end{aligned}
$$

The loads applied on each microcrack surface are $-\sigma_{N(k)}^{M I(i)}$ and $-\tau_{T(k)}^{M I(i)}$ in subproblem (ii), $-\left(\sigma_{N(k)}^{M I(i i)}+\sigma_{N(k)}^{M I(i i i)}\right)$ and $-\left(\tau_{T(k)}^{M I(i i)}+\tau_{T(k)}^{M I(i i i)}\right)$ in subproblem (iv), $-\left(\sigma_{N(k)}^{M I(i v)}+\sigma_{N(k)}^{M I(v)}\right)$ and $-\left(\tau_{T(k)}^{M I(i v)}+\tau_{T(k)}^{M I(v)}\right)$ in subproblem (vi), etc. The SIFs at the tip of the microcrack may be written as 


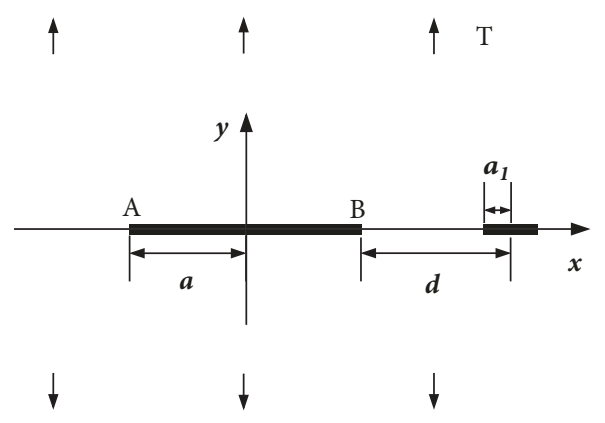

(a)

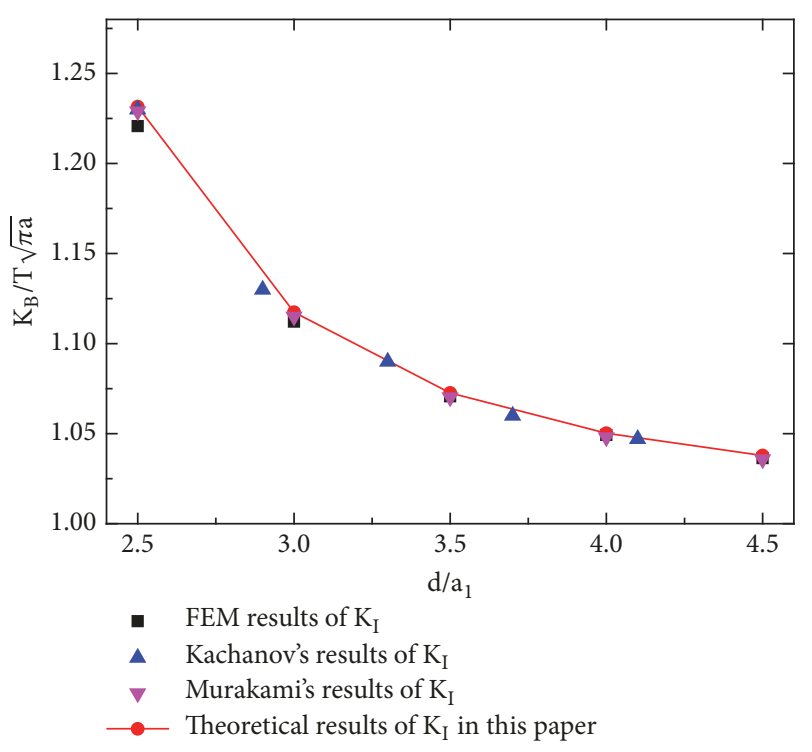

(b)

Figure 4: (a) Two collinear cracks under tensile load; (b) $K_{B} / T \sqrt{\pi \mathrm{a}}$ versus $d / a_{1}$ for $a / a_{1}=1$ under tensile load.

$$
\begin{gathered}
K_{I(k)}^{M I}=\int_{-a_{k}}^{a_{k}} \frac{-\left[\sigma_{N(k)}^{M I(i)}+\sigma_{N(k)}^{M I(i i)}+\sigma_{N(k)}^{M I(i i)}+\sigma_{N(k)}^{M I(i v)}+. .\right]}{\sqrt{\pi a_{k}}}\left(\frac{a_{k}+x_{k}}{a_{k}-x_{k}}\right)^{1 / 2} d x_{k} \\
K_{I I(k)}^{M I}=\int_{-a_{k}}^{a_{k}} \frac{-\left[\tau_{T(k)}^{M I(i)}+\tau_{T(k)}^{M I(i i)}+\tau_{T(k)}^{M I(i i i)}+\tau_{T(k)}^{M I(i v)}+. .\right]}{\sqrt{\pi a_{k}}}\left(\frac{a_{k}+x_{k}}{a_{k}-x_{k}}\right)^{1 / 2} d x_{k}
\end{gathered}
$$

\section{Results and Discussion}

In order to understand the macrocrack propagating and the microcracks getting together, it is designed to simulate the damage at the macrocrack tip that three distributions of microcracks as chained arranged microcracks, reversechained arranged microcracks and randomly oriented microcracks. At the first, the correctness of the step-by-step procedure should be verified.

3.1. Computational Accuracy Verification. An infinite plane containing two collinear cracks with the same length under mode I load is considered in Figure 4(a), where $a / a_{1}=1$. Crack $A B$ may be considered as the macrocrack in Section 2. The variation of normalized SIF $K_{B} / T \sqrt{\pi \mathrm{a}}$ at crack tip B versus distance $d / a_{1}$ is depicted in Figure $4(\mathrm{~b})$. The results obtained by the proposed theoretical method are in good agreement with the corresponding theoretical results $[29,30]$ and FEM results. The finite element model is established by ANSYS 12.0. It is observed that the normalized SIF at the crack tip B increase with the decrease of the distance between cracks.
3.2. Interaction between a Macrocrack and a Cluster of Chained Arranged Microcracks. It is considered that an infinite plane containing a macrocrack of length $2 a$ and a cluster of chained arranged microcracks of length $2 a_{k}(k=$ $1,2, \ldots, 14)$ as shown in Figure 5. The uniform tensile load $\mathrm{T}$ is applied at infinity perpendicular to the macrocrack. The length of each microcrack is taken as $a_{k}=0.1 a$. The fourteen microcracks are symmetrical about the $x$-axis. Some configurations of microcrack 1 to microcrack 7 (as in Figure 2) are given in Tables 1-3.

The nondimensional parameters for the macrocrack and each microcrack are taken as

$$
\begin{aligned}
K^{M A} & =T \sqrt{\pi a} \\
K^{M I} & =T \sqrt{\pi a_{k}} \quad(k=1, \ldots, n)
\end{aligned}
$$

In Figure 6, (+1) and (-1) represent the right and left crack tips respectively, where $d_{1}$ may be considered as the distance parameter between the macrocrack and each microcrack, and $\beta_{1}$ may be considered as the inclination parameter of the cluster of microcracks from the presupposed relation of each crack angle in Table 3. 


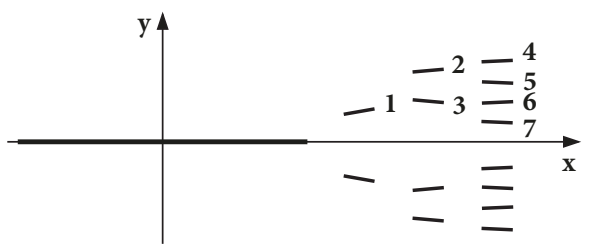

Figure 5: An infinite plane with a macrocrack and fourteen microcracks.

Due to the influence of microcracks, all values of $K_{I} / K^{M A}$ at the macrocrack tip are greater than 1 , all values of $K_{I I} / K^{M A}$ at the macrocrack tip are not equal to zero. In Figure 6(a), when $\beta_{1}$ is lower than 20 degrees, the strong amplification effects (which means the normalized SIF is larger than 1, the interaction effect between cracks enlarges the SIF at the crack tip) are observed at the macrocrack tip. The SIF decreases with the increase of inclination parameter $\beta_{1}$ and distance parameter $d_{1}$. On the contrary, shielding effect means the normalized SIF is less than 1, and the interaction effect between cracks decreases the SIF at the crack tip. In Figure $6(\mathrm{~b})$, the normalized $K_{I I} / K^{M A}$ is small relative to the normalized $K_{I} / K^{M A}$ and its influence is very small.

Due to the symmetry, only the normalized SIF over the $x$ axis is shown in Figure 7, where $d_{1}=2$. In Figure 7(a), when $\beta_{k}$ is between -30 and 20 degrees, the value of SIF is near or over 1, that means the microcracks act an amplification effect each other, and this is due to the interaction effect among microcracks. When $\beta_{k}$ is more than 20 degrees, a change from the amplification to the shielding occurs.

The results in Figure 7(b) show the SIF begins to decline from the peak when $\beta_{k}$ is more than 20 degrees. Moreover, the normalized $K_{I} / K^{M I}$ and $K_{I I} / K^{M I}$ of the microcracks have the same order of magnitude; it means that the microcracks are mixed type crack of mode I and mode II.

3.3. Interaction between a Macrocrack and a Cluster of ReverseChained Arranged Microcracks. An infinite plane containing a macrocrack of length $2 a$ and a cluster of reverse-chained arranged microcracks of length $2 a_{k}(k=1,2, \ldots, 14)$ is considered in Figure 8. The tensile stress $\mathrm{T}$ is applied at infinity perpendicular to the macrocrack. The length of each microcrack is taken as $a_{k}=0.1 a$. Some configurations of microcracks (as in Figure 2) are given in Tables 4-6.

The results in Figure 9 show that the effect of distance on SIF at the macrocrack tip has a similar behavior to Figure 6; it will be getting weaker as the distance between microcrack and macrocrack increases. However, the value of SIF is larger than that in Figure 6, due to the more concentrated microcracks in front of the macrocrack tip.

The normalized SIF at the microcrack tip over the $x$-axis is shown in Figure 10, where $d_{1}=2$. The greater the number of microcracks at the macrocrack tip, the bigger the value of normalized $K_{I} / K^{M I}$. The microcracks are mixed type crack of mode I and mode II because of the normalized $K_{I} / K^{M I}$ and $K_{I I} / K^{M I}$ of the microcracks being the same order of magnitude.
TABLE 1: The direction angle $\theta_{k}(k=1, \ldots, 7)$ of each microcrack.

\begin{tabular}{lcccccc}
\hline$\theta_{1}\left({ }^{\circ}\right)$ & $\theta_{2}\left({ }^{\circ}\right)$ & $\theta_{3}\left({ }^{\circ}\right)$ & $\theta_{4}\left({ }^{\circ}\right)$ & $\theta_{5}\left({ }^{\circ}\right)$ & $\theta_{6}\left({ }^{\circ}\right)$ & $\theta_{7}\left({ }^{\circ}\right)$ \\
\hline 20 & 25 & 15 & 20 & 15 & 10 & 5 \\
\hline
\end{tabular}

TABLE 2: The distance $d_{k}(k=1, \ldots, 7)$ between the macrocrack and each microcrack.

\begin{tabular}{lcc}
\hline$d_{1}$ & $d_{2,3}$ & $d_{4,5,6,7}$ \\
\hline$d_{1}$ & $d_{1}+3$ & $d_{1}+6$ \\
\hline 2 & 5 & 8 \\
3 & 6 & 9 \\
4 & 7 & 10 \\
5 & 8 & 11 \\
\hline
\end{tabular}

TABLE 3: The inclination angle $\beta_{k}(k=1, \ldots, 7)$ of each microcrack.

\begin{tabular}{lcccccc}
\hline$\beta_{1}\left({ }^{\circ}\right)$ & $\beta_{2}\left(^{\circ}\right)$ & $\beta_{3}\left(^{\circ}\right)$ & $\beta_{4}\left(^{\circ}\right)$ & $\beta_{5}\left(^{\circ}\right)$ & $\beta_{6}\left({ }^{\circ}\right)$ & $\beta_{7}\left({ }^{\circ}\right)$ \\
\hline$\beta_{1}$ & $\beta_{1} / 2$ & $-\beta_{1} / 2$ & $\beta_{1} / 4$ & $-\beta_{1} / 4$ & $\beta_{1} / 4$ & $-\beta_{1} / 4$ \\
\hline 10 & 5 & -5 & 2.5 & -2.5 & 2.5 & -2.5 \\
20 & 10 & -10 & 5 & -5 & 5 & -5 \\
30 & 15 & -15 & 7.5 & -7.5 & 7.5 & -7.5 \\
40 & 20 & -20 & 10 & -10 & 10 & -10 \\
50 & 25 & -25 & 12.5 & -12.5 & 12.5 & -12.5 \\
\hline
\end{tabular}

TABLE 4: The direction angle $\theta_{k}(k=1, \ldots, 7)$ of each microcrack.

\begin{tabular}{lcccccc}
\hline$\theta_{1}\left({ }^{\circ}\right)$ & $\theta_{2}\left({ }^{\circ}\right)$ & $\theta_{3}\left({ }^{\circ}\right)$ & $\theta_{4}\left({ }^{\circ}\right)$ & $\theta_{5}\left({ }^{\circ}\right)$ & $\theta_{6}\left({ }^{\circ}\right)$ & $\theta_{7}\left({ }^{\circ}\right)$ \\
\hline 40 & 30 & 20 & 10 & 15 & 10 & 5 \\
\hline
\end{tabular}

TABLE 5: The distance $d_{k}(k=1, \ldots, 7)$ between the macrocrack and each microcrack.

\begin{tabular}{lcc}
\hline$d_{1,2,3,4}$ & $d_{5,6}$ & $d_{7}$ \\
\hline$d_{1,2,3,4}$ & $d_{1,2,3,4}+3$ & $d_{1,2,3,4}+6$ \\
\hline 2 & 5 & 8 \\
3 & 6 & 9 \\
4 & 7 & 10 \\
5 & 8 & 11 \\
\hline
\end{tabular}

TABLE 6: The inclination angle $\beta_{k}(k=1, \ldots, 7)$ of each microcrack.

\begin{tabular}{lcccccc}
\hline$\beta_{1}\left({ }^{\circ}\right)$ & $\beta_{2}\left({ }^{\circ}\right)$ & $\beta_{3}\left({ }^{\circ}\right)$ & $\beta_{4}\left({ }^{\circ}\right)$ & $\beta_{5}\left({ }^{\circ}\right)$ & $\beta_{6}\left({ }^{\circ}\right)$ & $\beta_{7}\left({ }^{\circ}\right)$ \\
\hline$\beta_{1}$ & $-\beta_{1}$ & $\beta_{1}$ & $-\beta_{1}$ & $2 \beta_{1}$ & $-2 \beta_{1}$ & $4 \beta_{1}$ \\
\hline 2.5 & -2.5 & 2.5 & -2.5 & 5 & -5 & 10 \\
5 & -5 & 5 & -5 & 10 & -10 & 20 \\
7.5 & -7.5 & 7.5 & -7.5 & 15 & -15 & 30 \\
10 & -10 & 10 & -10 & 20 & -20 & 40 \\
12.5 & -12.5 & 12.5 & -12.5 & 25 & -25 & 50 \\
\hline
\end{tabular}

3.4. Interaction between a Macrocrack and a Cluster of Randomly Distributed Microcracks. In this section, consider an infinite elastic plane containing a macrocrack and nine randomly distributed microcracks, subjected to uniform tensile load in direction $y$. Each microcrack is assigned a random orientation and a prospective microcrack site. Figure 11 shows the randomly distributed microcracks in front 


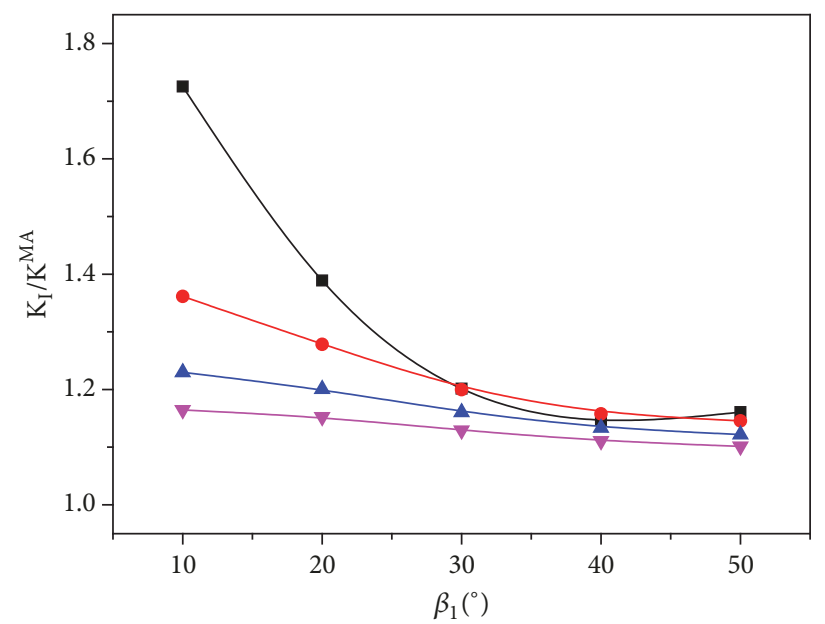

$(+1)$

$\rightarrow d_{1}=2$

$\sim d_{1}=4$

$\rightarrow d_{1}=5$

(a)

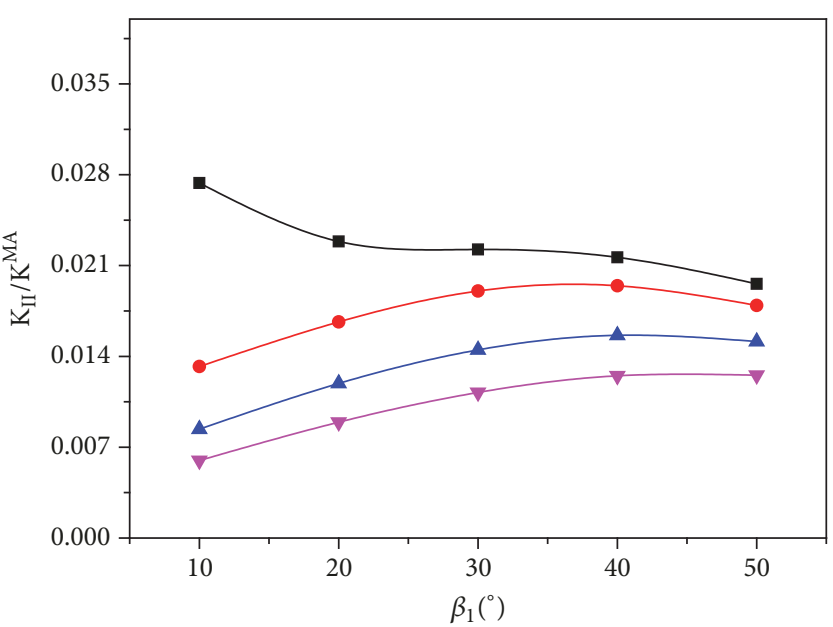

$(+1)$

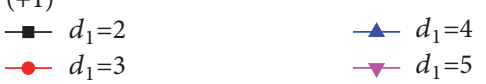

(b)

FIGURE 6: Normalized SIF at the right macrocrack tip versus inclination parameter $\beta_{1}$ for different distance parameter of $d_{1}$ between the cluster of microcracks and the macrocrack as in Tables 2-3. (a) $K_{I} / K^{M A}$; (b) $K_{I I} / K^{M A}$.

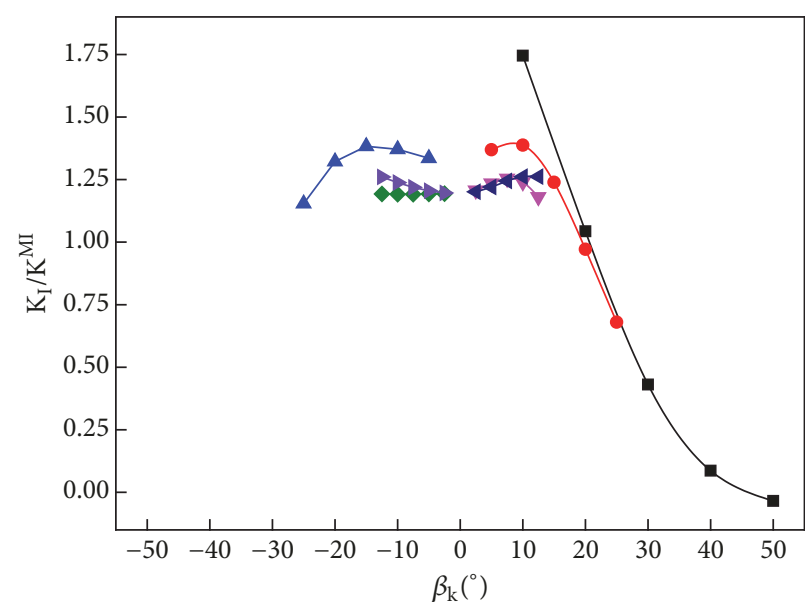

$(-1)$

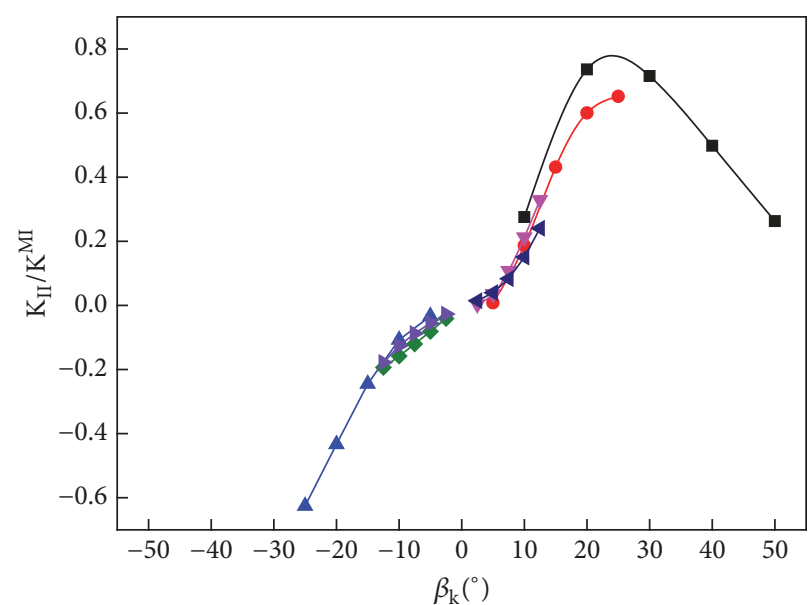

$(-1)$

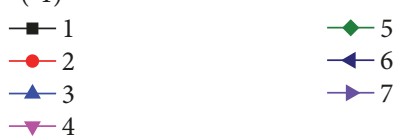

(b)

FIGURE 7: Normalized SIF at the left tip of each microcrack versus the inclination angle $\beta_{k}$ of each microcrack, and 1 to 7 represent the microcracks in Figure 5. (a) $K_{I} / K^{M I}$; (b) $K_{I I} / K^{M I}$.

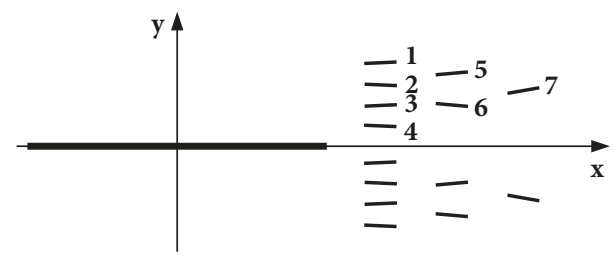

Figure 8: An infinite plane with a macrocrack and fourteen microcracks. of the macrocrack tip. Some configurations of microcracks (as in Figure 2) are given in Tables 7-9. The length of each microcrack is taken as $a_{k}=0.1 a(k=1,2, \ldots, 14)$. Four cases of inclination angles $\beta_{k}$ of microcrack are considered in Table 9.

With reference to (16), Tables 10 and 11 show the normalized SIF of macrocrack and microcracks; these SIFs are $K_{I}^{M A}=K_{I}^{M A} / K^{M A}, K_{I I}^{M A}=K_{I I}^{M A} / K^{M A}, K_{I(k)}^{M I}=K_{I}^{M I} / K^{M I}$, and $K_{I I(k)}^{M I}=K_{I I}^{M I} / K^{M I}$. 


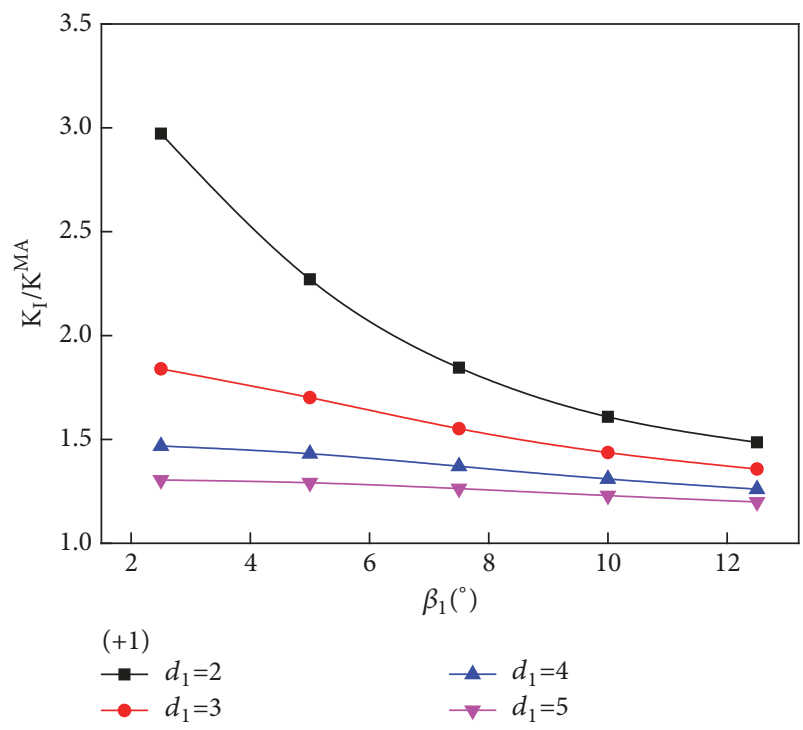

(a)

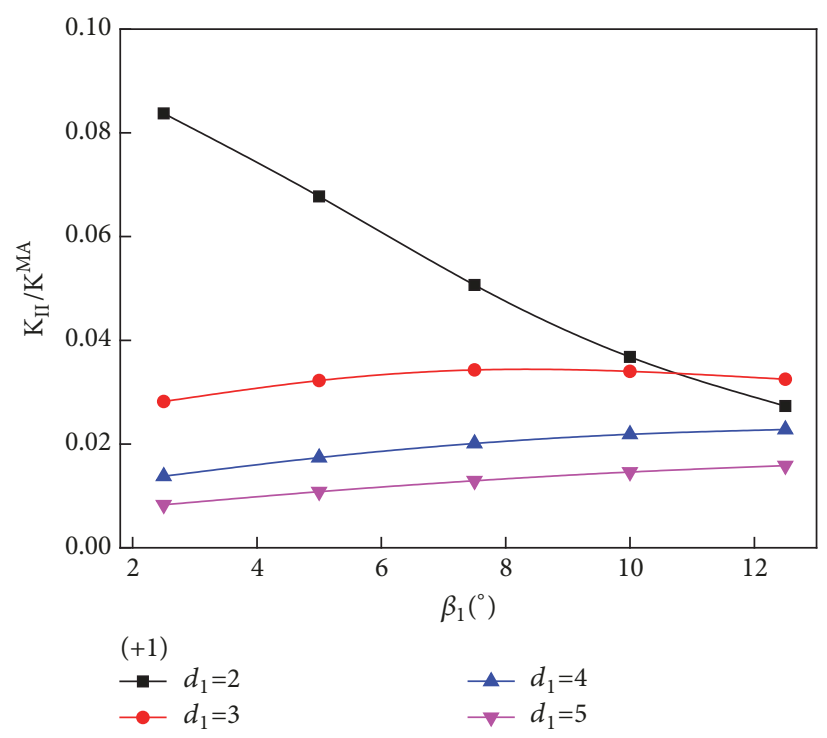

(b)

FIGURE 9: Normalized SIF at the right macrocrack tip versus inclination parameter $\beta_{1}$ for different distance parameter of $d_{1}$ between the cluster of microcracks and the macrocrack as in Table 5. (a) $K_{I} / K^{M A}$; (b) $K_{I I} / K^{M A}$.

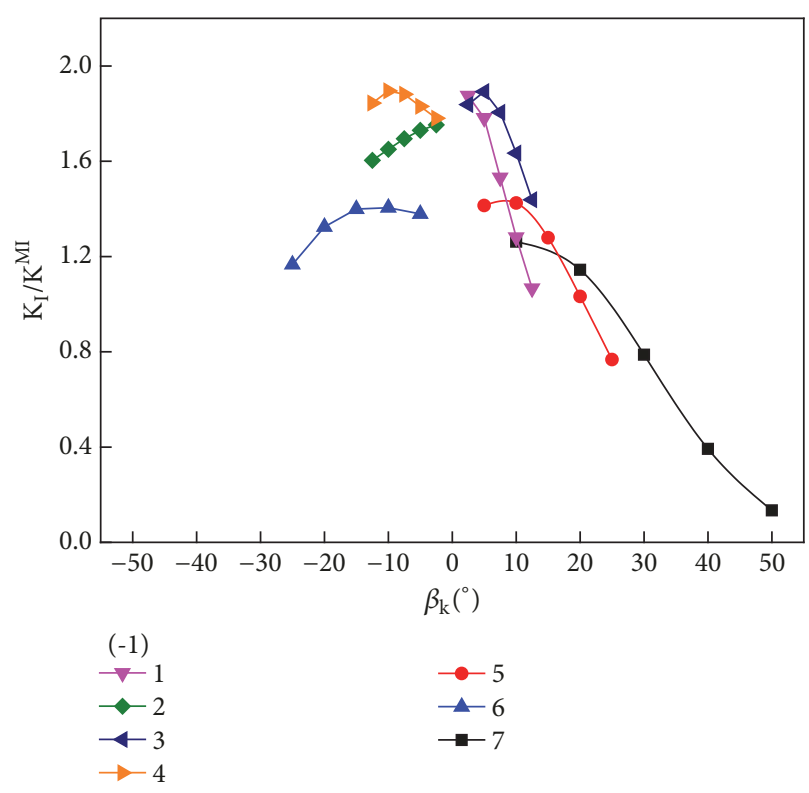

(a)

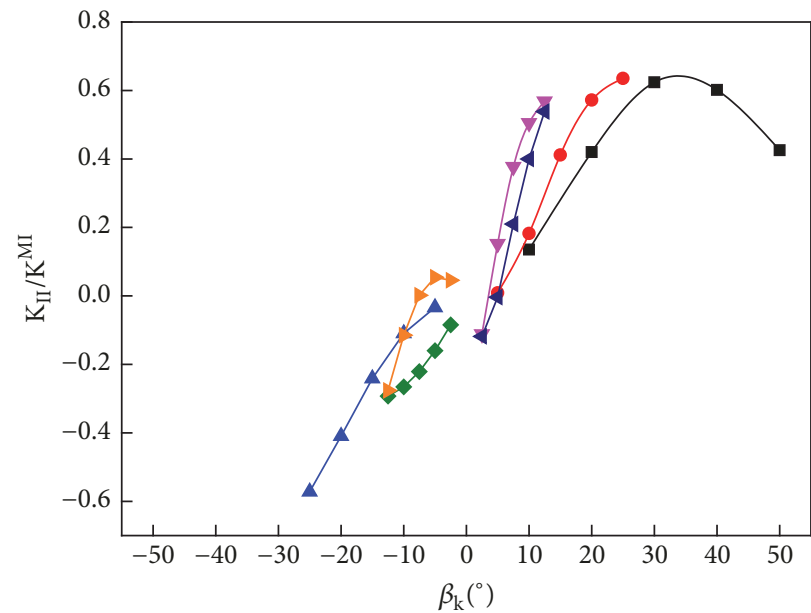

$(-1)$

$\rightarrow-1$
$\rightarrow-2$
$\rightarrow-3$

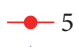

$-6$

$\rightarrow-7$

(b)

FIGURE 10: Normalized SIF at the left tip of each microcrack versus the inclination angle $\beta_{k}$ of each microcrack, and 1 to 7 represent the microcracks in Figure 9. (a) $K_{I} / K^{M I}$; (b) $K_{I I} / K^{M I}$.

TABLE 7: The direction angle $\theta_{k}(k=1, \ldots, 9)$ of each microcrack.

\begin{tabular}{ccccccccc}
\hline$\theta_{1}\left(^{\circ}\right)$ & $\theta_{2}\left(^{\circ}\right)$ & $\theta_{3}\left({ }^{\circ}\right)$ & $\theta_{4}\left({ }^{\circ}\right)$ & $\theta_{5}\left({ }^{\circ}\right)$ & $\theta_{6}\left({ }^{\circ}\right)$ & $\theta_{7}\left({ }^{\circ}\right)$ & $\theta_{8}\left(^{\circ}\right)$ & $\theta_{9}\left({ }^{\circ}\right)$ \\
\hline 80 & 60 & 10 & 30 & 0 & -45 & -20 & -70 & -90 \\
\hline
\end{tabular}

The results show the microcracks are mainly mixed type crack of mode I and mode II. Compared to the two chained arranged microcracks as discussed above, the macrocrack
TABLE 8: The distance $d_{k}(k=1, \ldots, 9)$ between the macrocrack and each microcrack.

\begin{tabular}{ccccccccc}
\hline$d_{1}$ & $d_{2}$ & $d_{3}$ & $d_{4}$ & $d_{5}$ & $d_{6}$ & $d_{7}$ & $d_{8}$ & $d_{9}$ \\
\hline 3.5 & 2.5 & 4.5 & 5.5 & 3.5 & 2.5 & 3.5 & 4.5 & 5.5
\end{tabular}

was affected little by randomly distributed microcracks; the value of the normalized $K_{I}^{M A}$ even decreased (shielding, $\left.K_{I}^{M A}<1\right)$. In other words, the concentrated distributed 
TABLE 9: The inclination angle $\beta_{k}(k=1, \ldots, 9)$ of each microcrack.

\begin{tabular}{lccccccccc}
\hline case & $\beta_{1}\left(^{\circ}\right)$ & $\beta_{2}\left(^{\circ}\right)$ & $\beta_{3}\left({ }^{\circ}\right)$ & $\beta_{4}\left({ }^{\circ}\right)$ & $\beta_{5}\left({ }^{\circ}\right)$ & $\beta_{6}\left({ }^{\circ}\right)$ & $\beta_{7}\left({ }^{\circ}\right)$ & $\beta_{8}\left({ }^{\circ}\right)$ & $\beta_{9}\left({ }^{\circ}\right)$ \\
\hline 1 & 60 & 0 & 45 & -20 & -80 & -10 & -70 & 60 \\
2 & 0 & 70 & -60 & 90 & 10 & -45 & -30 & -20 \\
3 & -45 & 20 & 0 & 70 & -30 & 90 & -80 & 80 \\
4 & 90 & 45 & -70 & 0 & 20 & 30 & -60 & 80 \\
\hline
\end{tabular}

TABLE 10: The mode-I SIF at the right macrocrack tip and left microcrack tips 1 to 9.

\begin{tabular}{llllccccccc}
\hline case & $K_{I}^{M A}$ & $K_{I(1)}^{M I}$ & $K_{I(2)}^{M I}$ & $K_{I(3)}^{M I}$ & $K_{I(4)}^{M I}$ & $K_{I(5)}^{M I}$ & $K_{I(6)}^{M I}$ & $K_{I(7)}^{M I}$ & $K_{I(8)}^{M I}$ & $K_{I(9)}^{M I}$ \\
\hline 1 & 0.956 & 0.06 & 1.89 & 0.15 & 1.29 & 0.06 & 1.47 & 0.03 & 0 \\
2 & 0.995 & 1.63 & 0.06 & 0 & 0.08 & 1.52 & 0.03 & 0.58 & 0.64 & 0 \\
3 & 0.999 & 0.11 & 0.76 & 1.36 & 0.08 & 0.70 & 0.07 & 0.08 & 1.63 \\
4 & 0.971 & 0.05 & 0.02 & 0 & 1.37 & 1.23 & 0.83 & 0 & 0 \\
\hline
\end{tabular}

TABLE 11: The mode-II SIF at the right macrocrack tip and left microcrack tips 1 to 9.

\begin{tabular}{llccccccccc}
\hline case & $K_{I I}^{M A}$ & $K_{I I(1)}^{M I}$ & $K_{I I(2)}^{M I}$ & $K_{I I(3)}^{M I}$ & $K_{I I(4)}^{M I}$ & $K_{I I(5)}^{M I}$ & $K_{I I(6)}^{M I}$ & $K_{I I(7)}^{M I}$ & $K_{I I(8)}^{M I}$ & $K_{I I(9)}^{M I}$ \\
\hline 1 & 0.122 & 0.11 & -0.02 & 0.51 & -0.32 & 0.006 & -0.46 & -0.06 & 0.30 & 0.43 \\
2 & -0.015 & 0.26 & 0.06 & -0.23 & -0.09 & 0.13 & -0.38 & -0.71 & -0.63 & -0.18 \\
3 & -0.053 & -0.70 & 0.72 & -0.05 & 0.11 & -0.74 & -0.08 & -0.03 & 0.38 & 0.35 \\
4 & 0.086 & -0.16 & 0.34 & -0.06 & -0.09 & 0.56 & 0.85 & -0.16 & -0.13 & -0.55 \\
\hline
\end{tabular}

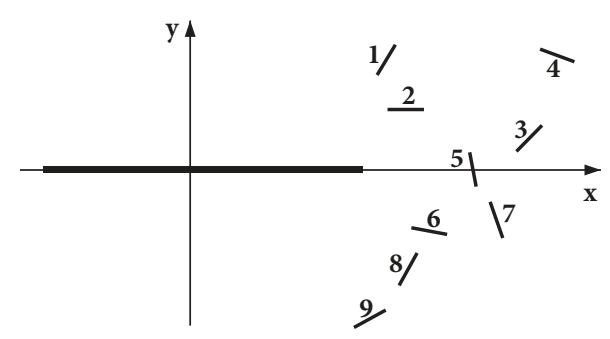

FIGURE 11: Nine randomly distributed microcracks in front of the macrocrack tip.

microcracks have greater effect on macrocrack than randomly distributed microcracks.

However, the interaction among microcracks does not decreased obviously in the four cases. The microcrack always acts an amplification effect $\left(K_{I}^{M I}>1\right)$ at about $0<\beta_{k}<10$ degrees; the microcrack acts a shielding effect $\left(K_{I}^{M I}<1\right)$ at about $\beta_{k}<-20$ and $\beta_{k}>30$ degrees.

Moreover, it may be predicted that the microcrack is more likely to be propagated when the inclination angle is between -10 and 10 degrees when the load increases or is subjected to fatigue load.

\section{Conclusions}

In this paper, a high efficient method based on Muskhelishvili's complex potential method is presented to study the interaction between a finite macrocrack and a cluster of microcracks. A step-by-step subproblem procedure is used to satisfy the stress boundary conditions on each crack surface. The stress intensity factor (SIF) of the macrocrack and the microcracks is obtained. With the comparison of these three kinds of damage configurations around the macrocrack tip, the interactions between a cluster of microcracks and a macrocrack and the interaction among microcracks are analyzed. Some conclusions can be summarized in the following:

(1) The chained and reverse-chained arranged microcracks increase SIF $K_{I}^{M A}$ of macrocrack at about $-50<\theta<50$ degrees, while the randomly distributed microcracks have little effects on the value of SIF $K_{I}^{M A}$ of macrocrack. In other words, the concentrated distributed microcracks have greater effect on macrocrack than randomly distributed microcracks.

(2) The effect of microcracks on SIF $K_{I}^{M A}$ at the macrocrack tip will be getting weaker as the distance between microcracks and macrocrack increases.

(3) For the chained and reverse-chained arranged microcracks, the SIF $K_{I(k)}^{M I}$ at the microcrack tip may be increased when the inclination angle of each microcrack is at about $-30<\beta_{k}<20$ degrees. When the inclination angle at about $\beta_{k}>20$ degrees, the shielding effect appears gradually.

(4) For the randomly distributed microcracks, the SIF $K_{I(k)}^{M I}$ at the microcrack tip may be increased when the inclination angle of each microcrack is at about $-20<\beta_{k}<$ 20 degrees.

(5) The microcracks are mixed type crack of mode I and mode II for the three distributions of microcracks.

As a future work on the subject, some guidelines may be given as follows.

The proposed theoretical method based on the Muskhelishvili's complex potential theory is a simple tool to solve crack problems, even for the complex cases such as a macrocrack interacting with a cluster of nine microcracks. It is worth noting that the proposed theoretical method is also appropriate to obtain the linear fracture mechanics solution 
of the pure mode II and mixed mode I/II crack problems, and the free traction conditions are also satisfied.

The results in the paper can provide some useful information to predict the fracture or damage behaviors in the macrocrack tip for the materials containing microcracks. What is more, different damage configurations may play an important role for crack shielding or enhancement. The SIF of the crack is affected by many factors, such as the number of microcracks, the distance between macrocrack and microcrack, and the orientation and direction of the microcrack. Thus, the analysis of different damage configurations at a macrocrack tip is important to materials design.

\section{Data Availability}

All the data used in the manuscript are available upon request by contact with the corresponding author (E-mail: xiaoyujiang8@sina.com).

\section{Conflicts of Interest}

The authors declare that they have no conflicts of interest.

\section{Acknowledgments}

The work is supported by the National Natural Science Foundation of China (11472230).

\section{References}

[1] Y. Gao, C. Zhang, X. Xiong, Z. Zheng, and M. Zhu, "Intergranular corrosion susceptibility of a novel Super $304 \mathrm{H}$ stainless steel," Engineering Failure Analysis, vol. 24, pp. 26-32, 2012.

[2] K. C. Hwang, S. Lee, and H. C. Lee, "Effects of alloying elements on microstructure and fracture properties of cast high speed steel rolls part II. Fracture behavior," Materials Science \& Engineering, vol. 254, no. 1-2, pp. 296-304, 1998.

[3] J. A. Ronevich, B. P. Somerday, and C. W. San Marchi, "Effects of microstructure banding on hydrogen assisted fatigue crack growth in X65 pipeline steels," International Journal of Fatigue, vol. 82, pp. 497-504, 2016.

[4] J. Song, C. Fan, H. Ma, L. Liang, and Y. Wei, "Crack deflection occurs by constrained microcracking in nacre," Acta Mechanica Sinica, vol. 34, no. 1, pp. 143-150, 2018.

[5] S.-X. Gong and H. Horii, "General solution to the problem of microcracks near the tip of a main crack," Journal of the Mechanics and Physics of Solids, vol. 37, no. 1, pp. 27-46, 1989.

[6] S. X. Gong and S. A. Meguid, "A general solution to the antiplane problem of an arbitrarily located elliptical hole near the tip of a main crack," International Journal of Solids and Structures, vol. 28, no. 2, pp. 249-263, 1991.

[7] S. X. Gong and S. A. Meguid, "Microdefect interacting with a main crack. A general treatment," International Journal of Mechanical Sciences, vol. 34, no. 12, pp. 933-945, 1992.

[8] M. Hori and S. Nemat-Nasser, "Interacting microcracks near the tip in the process zone of a macrocrack," Journal of the Mechanics and Physics of Solids, vol. 35, no. 5, pp. 601-629, 1987.

[9] W. Tian and Y. Chen, "Further investigation of interaction between interface macrocrack and parallel microcracks in bimaterial anisotropic solids," Acta Mechanica Sinica, vol. 15, no. 3, pp. 262-264, 1999.

[10] M. Kachanov, "A simple technique of stress analysis in elastic solids with many cracks," International Journal of Fracture, vol. 28, no. 1, pp. R11-R19, 1985.

[11] M. Kachanov, "Elastic solids with many cracks: A simple method of analysis," International Journal of Solids and Structures, vol. 23, no. 1, pp. 23-43, 1987.

[12] M. Kachanov, "Effective elastic properties of cracked solids: critical review of some basic concepts," Applied Mechanics Reviews, vol. 45, no. 8, pp. 304-335, 1992.

[13] V. Tamuzs, N. Romalis, and V. Petrova, Fracture of Solids with Microdefects, 2000.

[14] V. P. Tamuzs and V. E. Petrova, "On macrocrack-microdefect interaction," International Applied Mechanics, vol. 38, no. 10, pp. 1157-1177, 2002.

[15] V. Petrova and S. Schmauder, "Thermal fracture of a functionally graded/homogeneous bimaterial with system of cracks," Theoretical and Applied Fracture Mechanics, vol. 55, no. 2, pp. 148-157, 2011.

[16] V. Petrova and S. Schmauder, "Mathematical modelling and thermal stress intensity factors evaluation for an interface crack in the presence of a system of cracks in functionally graded/homogeneous bimaterials," Computational Materials Science, vol. 52, no. 1, pp. 171-177, 2012.

[17] V. Petrova, V. Tamuzs, and V. Petrova, "A survey of macromicrocrack interaction problems," Applied Mechanics Reviews, vol. 53, no. 5, pp. 117-146, 2000.

[18] R. G. Hoagland and J. D. Embury, "A Treatment of Inelastic Deformation Around a Crack Tip due to Microcracking," Journal of the American Ceramic Society, vol. 63, no. 7-8, pp. 404-410, 2010.

[19] I. Demir and H. M. Zbib, "A mesoscopic model for inelastic deformation and damage," International Journal of Engineering Science, vol. 39, no. 14, pp. 1597-1615, 2001.

[20] X. Yan, "Effective numerical approach for interaction of macrocrack with mircocracks," Chinese Journal of Theoretical \& Applied Mechanics, vol. 38, no. 1, pp. 119-124, 2006.

[21] X. Q. Yan, "Microcrack interacting with a finite main crack," Journal of Harbin Institute of Technology, vol. 40, no. 5, pp. 421430, 2006.

[22] X. Z. Xia, Q. Zhang, P. Z. Qiao, and L. I. Li-Juan, "Interaction between cracks and effect of microcrack zone on main crack tip," Applied Mathematics and Mechanics, vol. 31, no. 1, pp. 67$76,2010$.

[23] S. Rafoee, D. Gross, and T. Seelig, "The influence of microcrack nucleation on dynamic crack growth - a numerical study," Engineering Fracture Mechanics, vol. 71, no. 4, pp. 849-857, 2004.

[24] T. Seelig and D. Gross, "On the interaction and branching of fast running cracks - A numerical investigation," Journal of the Mechanics and Physics of Solids, vol. 47, no. 4, pp. 935-952, 1999.

[25] A. Brencich and A. Carpinteri, "Interaction of a main crack with ordered distributions of microcracks: a numerical technique by displacement discontinuity boundary elements," International Journal of Fracture, vol. 76, no. 4, pp. 373-389, 1986.

[26] X. Huang and B. L. Karihaloo, "Interaction of penny-shaped cracks with a half-plane crack," International Journal of Solids and Structures, vol. 30, no. 15, pp. 2117-2139, 1993.

[27] J.-P. Laures and M. Kachanov, "Three-dimensional interactions of a crack front with arrays of penny-shaped microcracks," International Journal of Fracture, vol. 48, no. 4, pp. 255-279, 1991. 
[28] N. I. Muskhelishvili, Some Basic Problems of the Mathematical Theory of Elasticity, Springer, Netherlands, 1975.

[29] M. Kachanov, "Elastic solids with many cracks and related problems," Advances in Applied Mechanics, vol. 30, no. 114, pp. 259-445, 1993.

[30] Y. Murakami and L. M. Keer, "Stress intensity factors handbook," Journal of Applied Mechanics, vol. 60, no. 4, article no. 1063, 1987. 


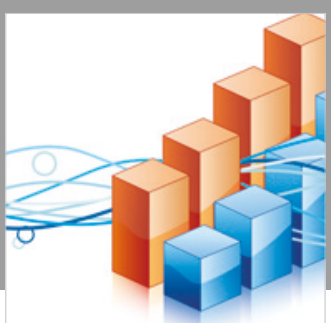

Advances in

Operations Research

\section{-n-m}
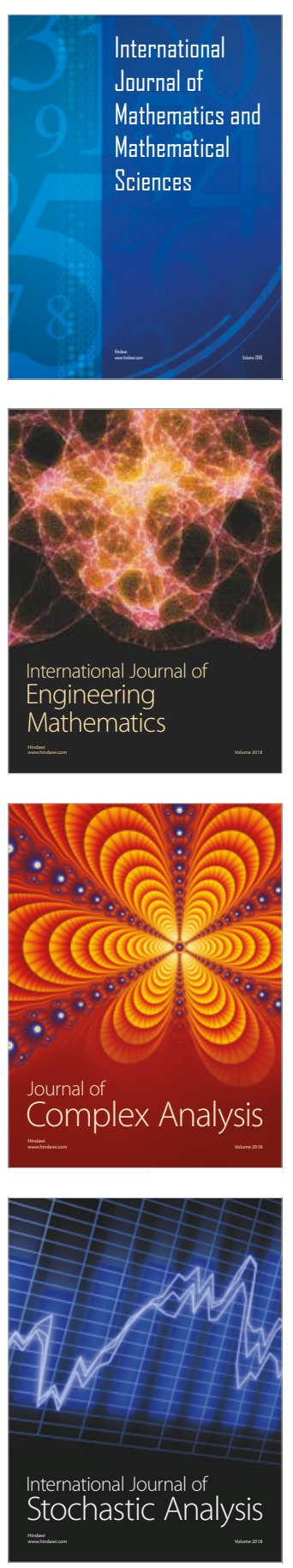
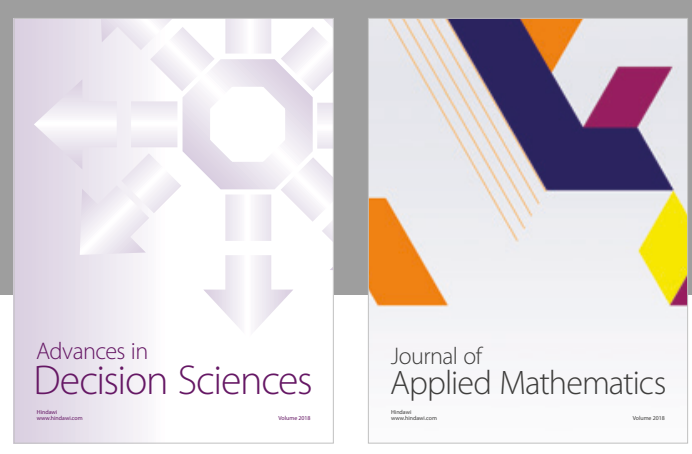

Journal of

Applied Mathematics
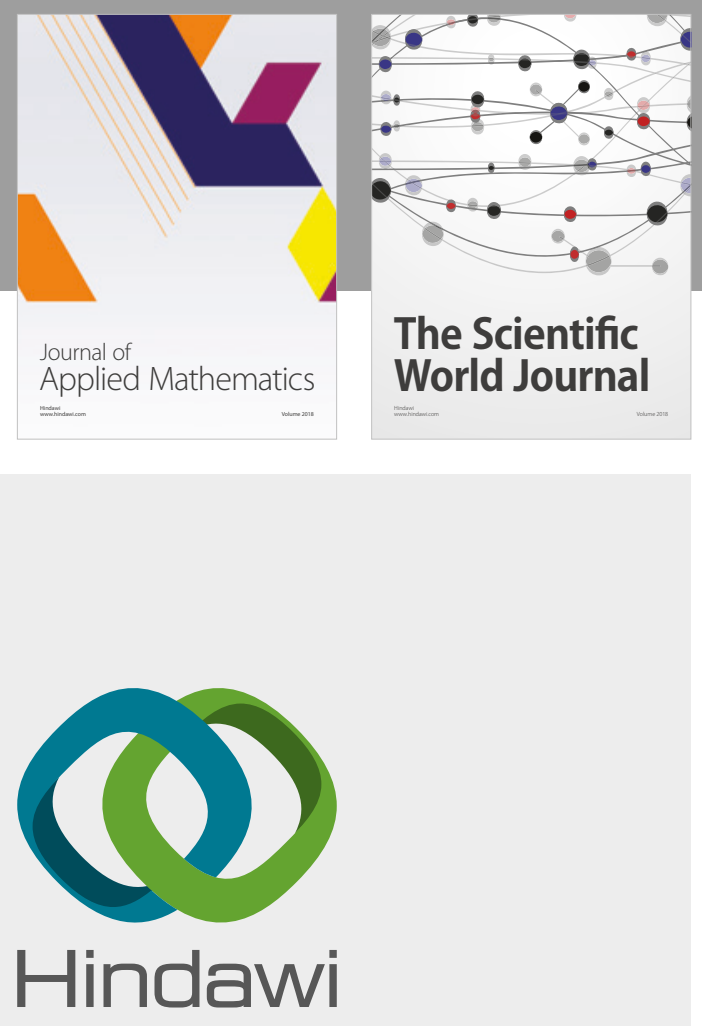

Submit your manuscripts at

www.hindawi.com

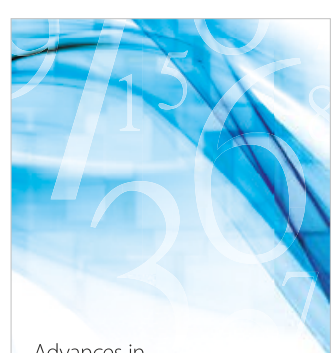

Advances in
Numerical Analysis
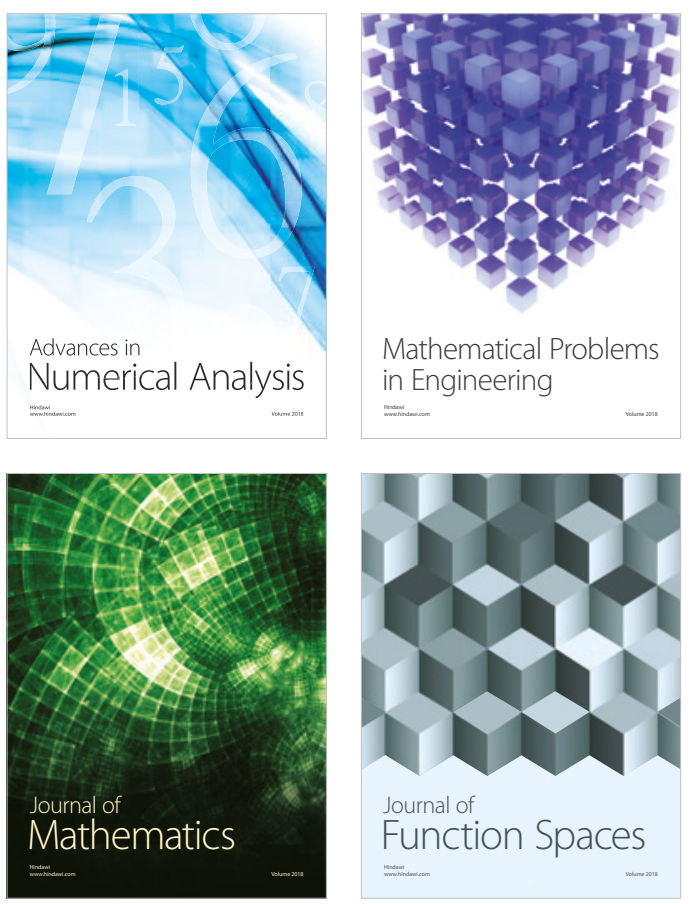

Mathematical Problems in Engineering

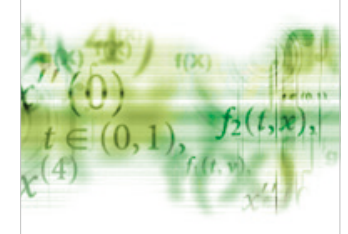

International Journal of

Differential Equations

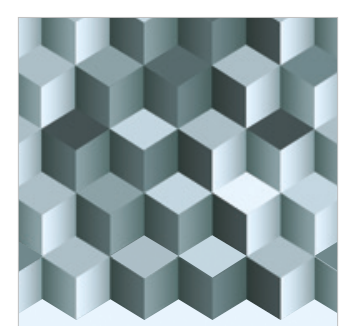

Journal of

Function Spaces

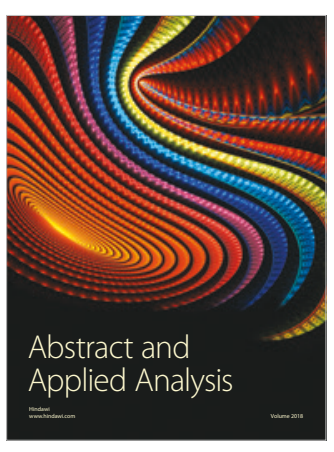

The Scientific

World Journal

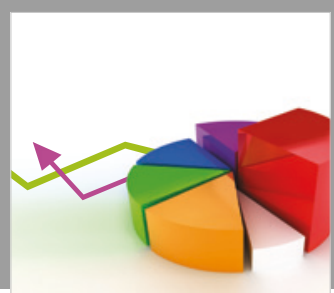

Journal of

Probability and Statistics
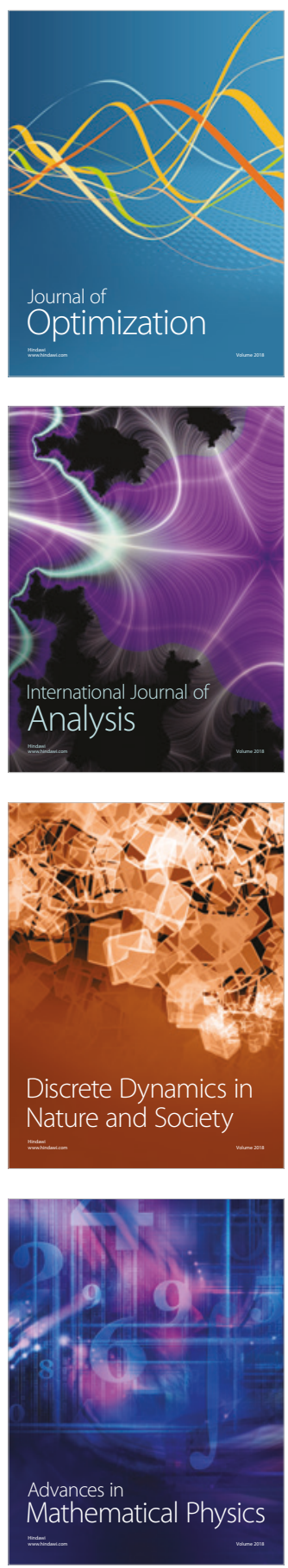